\title{
Is it Possible the Training of Prospective Memory to Enhance Activities of Daily Living and Executive Function in People with Mild Cognitive Impairment? A Single-blind Randomized Controlled Trial
}

\author{
Christina Agogiatou ${ }^{1}$, Nefeli Markou ${ }^{1}$, Eleni Poptsi ${ }^{1,2 *}$ and Magda \\ Tsolaki ${ }^{1,3}$ \\ ${ }^{1}$ Greek Association of Alzheimer Disease and Related Disorders (GAADRD), \\ Thessaloniki, Macedonia, Greece \\ ${ }^{2}$ Laboratory of Psychology, Section of Cognitive and Experimental Psychology, \\ Faculty of Philosophy, School of Psychology, Aristotle University of Thessaloniki \\ (AUTh), Macedonia, Greece \\ ${ }^{3} 1^{\text {st }}$ Department of Neurology, School of Medicine, Aristotle University of Thessaloniki \\ (AUTh), Macedonia, Greece \\ *Corresponding Author: Eleni Poptsi, Greek Association of Alzheimer's Disease and \\ Related Disorders (GAADRD), Thessaloniki, Macedonia, Greece.
}

\author{
Received: August 19, 2020 \\ Published: September 21, 2020 \\ (C) All rights are reserved by Eleni Poptsi., et al.
}

\begin{abstract}
Objective: The majority of elderly have deficits in Prospective Memory (PM) which are associated with difficulties in activities of daily living (ADL). Until today, only a few studies of prospective memory training have seemed to improve the cognitive functions, nevertheless this benefit has not been transferred to ADL. The aim of this study was the enhancement of executive functions, the generalization of training to other cognitive domains and the transference of these benefits to ADL.

Methods: From 156 patients with Mild Cognitive Impairment (MCI) who were examined at our Neurological Department and our day center, 102 people were randomly classified in one experimental Prospective Memory Group (PM/G) and one Control Group $(C / G)$, matched in age, gender, education, cognitive and functional status. Sixty-two out of them completed the study. The PM/G (n = 33) attended 48 one-hour sessions of PM training, twice a week for 6 months whereas the $C / G(n=29)$ did not participate at any intervention and were in a waiting list. Neuropsychological assessment including a test for PM was performed at baseline and after the end of the intervention.

Results: At the follow up, the PM/G outperformed the C/G in working memory $(p<0.05)$, in verbal fluency $(p<0.05)$, and in ADL ( $p<$ $0.05)$. At the end of the training the PM/G improved verbal memory $(p<0.05)$, verbal fluency $(p<0.05)$ and ADL $(p<0.05)$. The $\mathrm{C} / \mathrm{G}$ showed deterioration in verbal fluency $(p<0.05)$ whilst remained at the same performance level of ADL.

Conclusion: The PM intervention via the utilization of the decided tool, didn't succeed in improving PM, however it helped participants to improve executive functions, verbal memory and ADL.
\end{abstract}

Keywords: Prospective Memory; Cognitive Training; Cognitive Intervention

\section{Abbreviations}

PM: Prospective Memory; ADL: Activities of Daily Living; PM/G: Prospective Memory Group; C/G: Control Group; MCI: Mild Cog- nitive Impairment; aMCImd: Amnesic and Multiple Domain MCI; ChEIs: Cholinesterase Inhibitors 
Is it Possible the Training of Prospective Memory to Enhance Activities of Daily Living and Executive Function in People with Mild Cognitive Impairment? A Single-blind Randomized Controlled Trial

\section{Introduction}

Difficulties to remember to perform an intended action at an appropriate time in the future, are common in older adults, and in people with Mild Cognitive Impairment (MCI). Many of them experiences these changes, severely enough, to bring them to the medical doctor. A few examples concerning the aforementioned ability include remembering to deactivate the stove at the appropriate time in the future, or remembering to take their pills on time. The ability which helps people to remember to perform a planned action or intention in the appropriate time, is called Prospective Memory (PM) [1].

PM comprises two components, a) the prospective memory component which allows people to remember a task or an action that should be carried out at the right time, and b) the retrospective component which is related to the retrieval of the task/action $[2,3]$. During PM tasks a series of cognitive processes are involved in forming, retaining, initiating and executing an intention [1,2]. Specifically, the PM comprises four phases for the implementation of a prospective memory task. These are: (a) formation and encoding of intention and action; (b) intention- retention - the period during which the intention is retained, while ongoing activities are occurring [4]; (c) intention-initiation - the specific point in time at which execution of the intention is initiated; and (d) intention-execution - where the intended action is appropriately executed [1,5].

As obvious from the four phases of PM tasks, the component of $\mathrm{PM}$ is closely related to executive functions. Thus, when performing a PM task many executive function processes are engaged and activated such as working memory, shifting of attention, activation of the intention to act, monitoring, inhibition, strategies activation, and planning [6,7]. For example, when people have to perform a PM task, they have to continue performing the ongoing task, while at the same time they have to keep in their mind the prospective intention, to monitor the environment and also inhibit the inappropriate PM responses. According to literature, two types of PM targets exist, based on the PM cue: time - based when the intention executes at a specific time, and event - based when the intention executes after a specific cue appears [1].

Failures in PM tasks are common in general population and most of the time memory complaints in older adults refer to PM difficulties $[8,9]$. Older adults seem to perform more poorly than younger adults on laboratory PM settings and according to a meta - analysis there is a substantial age-related deficit in PM, where younger adults outperform older adults [10]. There is also evidence that people with MCI confront difficulties in PM [11,12]. It is suggested that PM is an early sign of memory failure in MCI people with a suspected pathology of Alzheimer's Disease (AD) and is a more pronounced deficit than retrospective memory [13,14]. Although PM is strongly associated with daily functioning and intact function of PM is crucial for a successful independent living, it is overlooked in memory training programs. Moreover, even though some evidence exists that PM training is effective for people with neurocognitive disorders [15], the effectiveness of PM training, specifically in people with $\mathrm{MCI}$, in other cognitive domains such as working memory, episodic memory or verbal fluency, is not yet evaluated. The aforementioned abilities seem to have a strong relation with executive functions. For example, damage to frontal brain areas is associated with poor performance in verbal fluency tasks $[16,17]$, whilst memory strategies are the prerequisite for successful episodic verbal learning [18]. On the other hand, working memory is a significant predictor of complex prospective memory initiation $[19,20]$.

\section{Conceptual framework}

Considering the relation between PM deficits, independent living and increased risk of developing dementia [21,22], we designed a cognitive training program of PM for people with amnesic and multiple domain MCI (aMCImd). Two approaches in cognitive training programs exist: 1) process - based training, aiming to restore or ameliorate the underlying impairment through repetitive practice of PM activities, 2) strategy - based training, aiming to compensate the deficits in underlying cognitive processes. Previous interventions used external compensatory strategies, such as calendars, agendas, timers, etc. and most of the tasks were not naturalistic. These showed improvements on specific tasks trained during the interventions, but failed to generalize this outcome in other cognitive abilities or transfer the benefit in ADL [23-25]. Based on the above results and to the evidence that PM is essential for independent living which is related with executive functions, we decided to design a new PM training program by using visual imagery as a learning strategy [26,27], towards improving executive function components, such as working memory and verbal fluency, as well as learning abilities and ADL.

Therefore, the aim of this single-blind randomized controlled trial was to evaluate the effectiveness of the proposed PM training program in community-dwelling and Greek-speaking people with 
Is it Possible the Training of Prospective Memory to Enhance Activities of Daily Living and Executive Function in People with Mild Cognitive Impairment? A Single-blind Randomized Controlled Trial

aMCImd, aged from 60 to 80 years old. The basic hypotheses were formulated as:

- $\quad$ The $P M / G$ at the end of the training sessions would present significant differences compared to $\mathrm{C} / \mathrm{G}$ in $\mathrm{PM}$ and in executive function components, such as in working memory and verbal fluency, since PM is closely related with executive functions.

- The PM/G after the end of the sessions would present improvement in abilities related with executive functions and $\mathrm{ADL}$ as well, as a result of the generalization of the primary cognitive benefit in daily function and because of the association between PM with executive functions and ADL.

- The $C / G$ after a period of 6 months would show stabilization of cognitive and functional performance or a mild deterioration.

\section{Materials and Methods}

\section{Participants}

This experimental study was carried out between September 2016 and June 2017 in the Day Care Centre of the Greek Association of Alzheimer Disease and Related disorders (Alzheimer Hellas), "Saint Helen". The inclusion criteria of this study were a) age more than 60 years, b) a diagnosis of aMCImd according to Petersen criteria [28], c) intact language skills, d) insight of cognitive problems, e) subjective complaints of people with MCI regarding difficulties in PM abilities, and f) diminished performance at the Royal Prince Alfred Prospective Memory Test (RPA ProMem) [29]. The exclusion criteria comprised a) severe depression/anxiety or behavioral problems such as aggressiveness and irritability, b) other neurological disorders such as epilepsy or stroke, c) uncontrolled hypertension and cardiovascular disease, d) pharmaceutical treatment with cholinesterase inhibitors (ChEIs), antipsychotic, anticholinergic drugs and benzodiazepines, and e) sensory deficits. Neurological examination, extensive neuropsychological and neuropsychiatric assessment, neuroimaging and blood tests were performed, in order to exclude other causes of MCI, such as MCI because of Parkinson, stroke, epilepsy or because of alcohol abuse etc., and the diagnosis was supported by a consensus of specialized health professionals.

The baseline design of the study comprised the recruitment of 102 people with aMCImd according to the inclusion and exclusion criteria that were presented above. Thus, 54 of them $(n=54)$ were randomly assigned in one experimental group in order to participate in the $\mathrm{PM}$ training $(\mathrm{PM} / \mathrm{G})$, while 48 people were randomly assigned to the control group $(C / G)(n=48)$. The $C / G$ didn't take part in any cognitive or other intervention in the Day Care centers of the Greek Association of Alzheimer Disease and Related Disorders, or in any other facility and recorded in a waiting list. Out of the 54 participants of the experimental group, 8 were excluded from the analysis because they missed more than five sessions, 6 withdraw from the study because of other medical reasons and 7 withdraw because they had difficulties to follow the trial instructions. Out of the 48 participants of the control group, 6 were lost to follow up, 4 withdraw the study for personal reasons, 4 refused the follow up because of family obligations, 2 were far away for the Day Care Center, and 3 had health issues (Figure 1). Therefore, only 62 older adults completed the follow-up and were considered for the analysis. The experimental groups comprised 12 men and 21 women, with a mean age of 69.27 years, $S D=7.17$, mean education of 12.93 years $(S D=4.24)$ and mean performance of 28.21, $S D=1.31$, at Mini Mental State Examination Test (MMSE). Concerning the control group, it consisted of 9 men and 20 women, with a mean age of 70.44 years, $\mathrm{SD}=7.65$, mean education of 13.10 years $\mathrm{SD}=3.76$, and a mean performance of 27.65, SD $=2.09$, at MMSE. Participants were matched according to age $(p=0.454)$, gender $\left(x^{2}=0.789\right)$ and education ( $p=0.929$ ) (Table 1$)$.

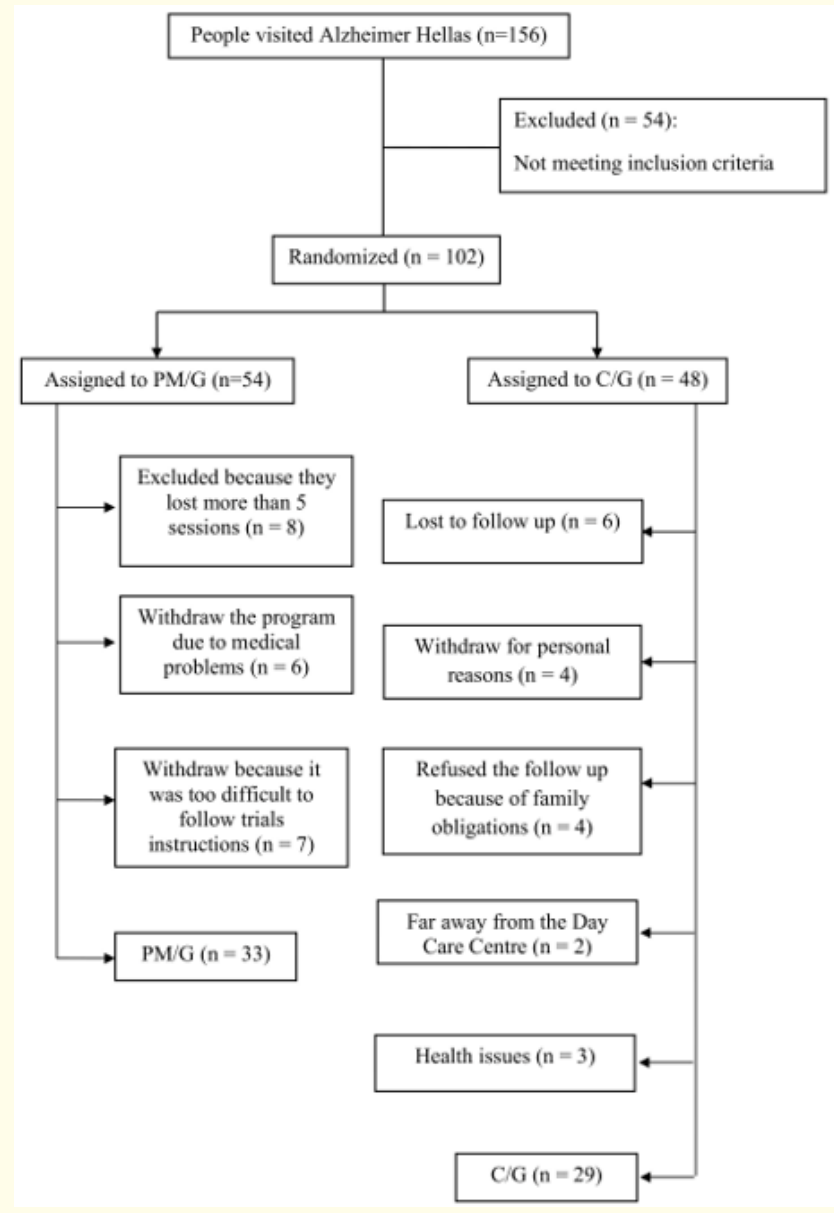

Figure 1: Chart flow of the sample. 
Is it Possible the Training of Prospective Memory to Enhance Activities of Daily Living and Executive Function in People with Mild Cognitive Impairment? A Single-blind Randomized Controlled Trial

\begin{tabular}{|l|c|c|c|}
\hline \multirow{2}{*}{$\begin{array}{l}\text { Demographic } \\
\text { Characteristics }\end{array}$} & \multicolumn{3}{|c|}{ Groups M (SD) } \\
\cline { 2 - 4 } $\begin{array}{l}\text { General Cognitive } \\
\text { and Functional } \\
\text { performance }\end{array}$ & $\mathbf{A} / \mathbf{G}$ & $\mathbf{P M} / \mathbf{G}$ & $\boldsymbol{p}$ \\
\hline Age & $70.44(7.65)$ & $\mathbf{N}=\mathbf{3 3}$ & \\
\hline Gender M/F $\left(\mathrm{x}^{2}\right)$ & $9 / 20$ & $12 / 21$ & 0.789 \\
\hline Years of education & $13.10(3.76)$ & $12.93(4.24)$ & 0.929 \\
\hline MMSE $\ddagger$ & $27.65(2.09)$ & $28.21(1.31)$ & 0.209 \\
\hline FRSSD-tot $\dagger$ & $3.82(1.87)$ & $3.90(1.68)$ & 0.857 \\
\hline FUCAS-tot $\dagger$ & $44.00(1.69)$ & $43.93(1.47)$ & 0.881 \\
\hline FUCAS-ac $\dagger$ & $6.20(.41)$ & $6.39(.60)$ & 0.168 \\
\hline RAVLT WM $\neq$ & $5.62(.40)$ & $6.12(.37)$ & 0.368 \\
\hline RAVLT-L $\ddagger$ & $10.24(2.65)$ & $11.36(2.24)$ & 0.076 \\
\hline FAS(X) $\ddagger$ & $9.20(2.79)$ & $10.24(2.78)$ & 0.146 \\
\hline FAS-tot $\neq$ & $10.94(3.00)$ & $11.56(3.05)$ & 0.429 \\
\hline RPA-tot $\neq$ & $8.84(2.80)$ & $9.36(2.29)$ & 0.448 \\
\hline
\end{tabular}

Table 1: Demographic characteristics of groups' participants.

M (SD): Mean (Standard Deviation); M/F: Male/Female; $x^{2}$ : Pearson's Chi square; C/G: Control Group; PM/G: Progressive Memory group; p: 0.05; ‡: More points/better performance; †: Less Points/ better performance; MMSE: Mini Mental State Examination; FRSSD: Functional Rating Scale for Dementia; FUCAS-tot: Functional Cognitive Assessment Scale-total score; RAVLT WM: Rey Auditory Verbal Learning Test/working memory; RAVLT L: Rey Auditory Verbal Learning Test/learning ability; FUCAS-ac: Accuracy of steps of FUCAS; FAS(X): Verbal Fluency/letter X; FAS(S): FAS-tot: Verbal Fluency, total score; RPA-tot: Prospective Memory Test total score.

\section{Randomization, allocation concealment and blinding}

In order to minimize selection bias, randomization and allocation concealment to the participants were used. The inclusion of participants in the study comprised computer-generated random numbers that were used for simple randomization (https://www. random.org/). Until the completion of the follow-up, only the project leader had access to the group allocation information. The independent evaluators were the same, both at the baseline and follow-up assessment and they were blinded with respect to group allocation. The participants of both groups of the study signed that they were aware that they could withdraw their consent at any time, without their statutory rights or medical or psychological care being affected. After the baseline assessment, eligible and consenting people with aMCImd were randomized in each group.
The intervention

\section{Prospective memory group (PM/G)}

The designed PM training program comprised three PM tasks in every session; a) an event - based task (non-focal PM task), b) a time - based task, and c) a combination task (the intention should be executed after a specific period of time and if a specific cue appeared). This specific structure was chosen, in order to include tasks similar to ADL. The tasks had internal and external levels of difficulty. As far as the internal levels of difficulty are concerned, the first task of the session was always simpler than the second, and the second was always simpler than the third. As far as the external level of difficulty is concerned, the training had three external levels of difficulty, involving increasing the time between the formation and the execution, in order to create a more complex intention, resulting in more complex tasks. This way, the intervention was adjusted to the participants' needs. The external level of difficulty changed for each participant after the completion of 16 sessions, under the condition that each participant could correctly complete all the previous tasks.

All tasks were naturalistic in order for the trainees to transfer the cognitive benefit to their ADL. The ongoing tasks included occupation with puzzles, watching videos, listening to music, doing handcrafts, reading newspapers, making shopping lists, etc. Intentions included interaction with other participants or employees of the day care center, such as message delivering, objects transferring etc. Ongoing tasks were different for every session, in order to maintain participants' motivation and to avoid systematically practicing any cognitive ability.

Each task included four phases of PM, similar to ADL. The structure of the sessions was constant, and the participants were training the PM components repetitively in the same way. The first session was a preparatory one, it was individualized and was divided into two parts: a) an informative one, where participants were informed regarding the PM components and their association with $\mathrm{ADL}$, and b) a strategy teaching one, where the participants were trained in specific strategies for the implementation of the cognitive tasks during the sessions of PM. The strategies taught were the implementation - intention, the encoding and the visual imagery. Besides the fact that the first strategy seems to enhance PM according to literature, it does not prevent PM decline in most cognitively - demanding PM tasks [30]. Therefore, the trainers were encouraged to use the strategy of visual imagery, which comprise the visu- 
Is it Possible the Training of Prospective Memory to Enhance Activities of Daily Living and Executive Function in People with Mild Cognitive Impairment? A Single-blind Randomized Controlled Trial

alization of all steps that trainers have to follow in order to execute an intention, both during the sessions and during their daily lives.

The trainees attended forty-eight (48) sessions in a period of 6 months. According to schedule they had to visit the day center twice a week for an hour. The time duration of six (6) months was chosen since studies have shown its adequacy to allow changes in brain that can be associated with cortical reorganization and plasticity [31]. The sessions always took place in the morning and each group comprised seven (7) participants. The PM program was administered by expert psychologists trained in cognitive interventions. A detailed session of the PM training is presented at annex 1.

\section{Control group $(\mathrm{C} / \mathrm{G})$}

The control group did not participate in any cognitive or other psychotherapeutic or physical training program in the day care center. They were people that for personal reasons they could not attend at the specific time period any cognitive intervention, therefore, they agreed to be in a waiting list for taking part in the training sessions 6 months later. Therefore, they were informed and agreed that they had to be followed up after 6 months. A trained secretary of the Day Care Center uptook the task to communicate with them by a phone call after 6 months.

The study was approved by the Scientific and Ethics Committee of Alzheimer Hellas (decision number: 25/21-06-2006). All groups underwent a baseline neuropsychological assessment 2 weeks prior the program's initiation and 6 months later.

\section{Neuropsychological assessment}

An extensive neuropsychological battery was chosen in order to assess the effectiveness of the interventions. Neuropsychological assessment included a battery of psychometric tests that comprised: a) the Mini Mental State Examination (MMSE) for the assessment of global cognitive performance and the stage of the disease [32,33], b) the Functional Rating Scale for Symptoms of Dementia (FRSSD) which assesses the caregiver's opinion regarding the participants daily function [34], c) the Functional Cognitive Assessment Scale (FUCAS) [35], (assesses executive function abilities such as planning, prospective memory, goal maintenance) in 5 aspects of daily life, d) the Rey Auditory Verbal Learning Test (RAVLT) for verbal episodic memory (learning ability) and working memory [36,37], e) the total score of the phonological Verbal Fluency Test - FAS and separate the score of Greek letter chi (X) for verbal fluency [38], and f) the Prospective Memory Test (RPAProMem) [29], towards assessing PM abilities.

\section{Statistical analysis}

The statistical analysis was performed with the IBM SPSS Statistics for Windows, Version 23.0 (Armonk, NY: IBM Corp 23.0) [39]. Demographic characteristics such as age, gender and education were analyzed at baseline using a Univariate analysis (ANOVA) and Chi square test, when necessary. In order to determine whether any significant difference between all groups at time of assessment existed, a mixed measure ANOVA 2 (between subjects' factor: group: PM/G-C/G x 2) (within subjects' factor: time of assessment: baseline-follow up) was used. Multivariate analysis of variance (MANOVA) was also used in order to examine the between-group differences at the follow-up. In order to assess the effect of PM training for the experimental group and the course of $\mathrm{C} / \mathrm{G}$ during time, repeated measures analysis of variance (ANOVA) were used. Bonferroni or any other correction was not used because of the diminished cognitive test, therefore, the statistical level of difficulty was set at $p=0.050$.

\section{Results}

\section{The effect of group and assessment time}

Baseline measurements showed that there were no statistically significant differences between all groups concerning demographic characteristics such as age $(p=0.454)$, gender $\left(x^{2}=0.789\right)$ or education ( $p=0.929$ ), in all cognitive and functional tests (Table 1 ).

The effect of group and assessment time in executive function

Regarding the total score of FUCAS, the MANOVA having the group as the independent variable and performance in two periods of assessment as the dependent one, showed that at the follow up there was a significant interaction effect $F(1,60)=6.29, p<0.05$, $\eta^{2} .09$. The PM/G outperformed the $\mathrm{C} / \mathrm{G}$ in the total FUCAS score ( $p$ $=0.015$ ) (Table 2 ). The subsequent within group ANOVA (repeated measures) performed between baseline and follow-up assessment separately in each group, showed neither improvement nor deterioration regarding the two groups' performance on the above test.

As far as the specific subtasks of FUCAS are concerned, the MANOVA showed that there was a significant interaction effect on the accuracy of steps $F(1,62)=9.82, p<0.05, \eta^{2} .14$. The PM/G outperformed the $\mathrm{C} / \mathrm{G}$ in accuracy of steps ( = 0.003) (Table 2). The subsequent ANOVA's for repeated measures, showed neither improvement nor deterioration regarding the two groups' perfor- 
Is it Possible the Training of Prospective Memory to Enhance Activities of Daily Living and Executive Function in People with Mild Cognitive Impairment? A Single-blind Randomized Controlled Trial

107

mance on the above subtasks of FUCAS.

As far as the working memory is concerned, there was a significant interaction effect $\mathrm{F}(1,60)=4.38, p<0.05, \eta^{2} .03$ in working memory ability of the RAVLT (RAVLT-WM). The PM/G outperformed the $C / G(p=0.041)$. On the contrary a main interaction effect was not noticed. Repeated measures showed neither improvement nor deterioration regarding the two groups' performance on the aforementioned ability.

As far as the verbal fluency - letter chi (FAS- $X$ ) is concerned a significant interaction effect was not noticed. However, according to the MANOVA, there was a main effect $\mathrm{F}(1,60)=7.38, p<0.05$, $\eta^{2} .11$. The repeated measures of ANOVA performed between baseline and follow up assessment regarding the $P M / G$, showed that the $\mathrm{PM} / \mathrm{G}$ showed improvement in the above ability $\mathrm{F}(1,32)=5.70$ $p<0.05, \eta^{2} .15(p=0.023)$, while the $\mathrm{C} / \mathrm{G}$ showed deterioration $\mathrm{F}(1$, 28) $=5.69, p<0.05, \eta^{2} .16(\mathrm{p}=0.024)$.

As far as the total score of FAS (FAS-tot) in concerned, the MANOVA showed an interaction effect $\mathrm{F}(1,60)=7.38, p<0.05, \eta^{2}$ $=.11$. The PM/G outperformed the $\mathrm{C} / \mathrm{G}(p=0.027)$ in FAS-tot (Table 2 ). The subsequent ANOVA for repeated measures, showed neither improvement nor deterioration regarding the two groups' performance on the above subtasks of FAS-tot.

The effect of group and assessment time in verbal episodic memory

Regarding the learning ability of RAVLT (RAVLT-L), there was not a significant interaction effect. However, the MANOVA showed a significant main effect $F(1,60)=7.01, p<0.05, \eta^{2} .10$. The subsequent within group ANOVA performed between baseline and follow up assessment, separately in the two groups, showed that the $\mathrm{PM} / \mathrm{G}$ after the training sessions improved the above ability $\mathrm{F}(1$, $32)=8.45, p<0.05, \eta^{2} .20(p=0.007)$. The $C / G$ didn't show any improvement or deterioration on the above ability (Table 3 ).

\section{The effect of group and assessment time in ADL}

According to MANOVA a significant interaction effect regarding FRSSD was not noticed. However, there was a main effect of time of performance on the total score of $\operatorname{FRSSD~} \mathrm{F}(1,60)=9.26, p<0.05$, $\eta^{2} .13$. The repeated measures showed that after the prospective training the $\mathrm{PM} / \mathrm{G}$ improved on the above ability $\mathrm{F}(1,32)=4.41, p<$ $0.05, \eta^{2} .18(p=.0011)$. The $\mathrm{C} / \mathrm{G}$ showed neither improvement nor deterioration in the same test.

\begin{tabular}{|l|c|c|c|}
\hline \multirow{2}{*}{$\begin{array}{l}\text { Neuropsychological } \\
\text { Tests }\end{array}$} & \multicolumn{3}{|c|}{ Groups M (SD) } \\
\hline & C/G (n = 29) & PM/G (n = 33) & $p$ \\
\hline MMSE $\ddagger$ & $27.55(2.06)$ & $28.66(1.51)$ & 0.130 \\
\hline FRSSD-tot $\dagger$ & $3.13(2.13)$ & $3.18(1.70)$ & 0.936 \\
\hline FUCAS-tot $\dagger$ & $44.86(2.44)$ & $43.33(1.47)$ & 0.015 \\
\hline FUCAS-ac $\dagger$ & $6.37(.56)$ & $6.12(.33)$ & 0.003 \\
\hline RAVLT-WM $\ddagger$ & $5.48(2.02)$ & $6.96(2.29)$ & 0.041 \\
\hline RAVLT-L $\ddagger$ & $10.75(2.58)$ & $12.15(2.26)$ & 0.586 \\
\hline FAS $(X) \ddagger$ & $9.96(3.09)$ & $11.78(4.06)$ & 0.357 \\
\hline FAS-tot & $10.78(2.95)$ & $12.78(3.33)$ & 0.027 \\
\hline RPA-tot & $8.52(3.34)$ & $9.53(2.37)$ & 0.494 \\
\hline
\end{tabular}

Table 2: Differences in performance at the follow up, between the two groups.

M (SD): Mean (Standard Deviation); C/G: Control Group; PM/G: Progressive Memory group; p: 0.05; $\ddagger$ : More points/better performance; †: Less Points/ better performance; MMSE: Mini Mental State Examination; FRSSD: Functional Rating Scale for Dementia; FUCAS-tot: Functional Cognitive Assessment Scale-total score; RAVLT WM: Rey Auditory Verbal Learning Test/working memory; RAVLT L: Rey Auditory Verbal Learning Test/learning ability; FUCAS-ac: Accuracy of steps of FUCAS; FAS(X): Verbal Fluency/ letter X; FAS-tot: Verbal Fluency, total score; RPA-tot: Prospective Memory Test total score.

\begin{tabular}{|l|c|c|c|c|}
\hline \multirow{2}{*}{ Test } & Groups & $\begin{array}{c}\text { Baseline } \\
\text { M (SD) }\end{array}$ & $\begin{array}{c}\text { Follow-up } \\
\text { M (SD) }\end{array}$ & $\boldsymbol{p}$ \\
\hline \multirow{2}{*}{ FRSSD-tot $\dagger$} & $\mathrm{C} / \mathrm{G}$ & $3.82(1.87)$ & $3.13(2.13)$ & 0.089 \\
\cline { 2 - 5 } & $\mathrm{PM} / \mathrm{C}$ & $3.90(1.68)$ & $3.18(1.70)$ & 0.011 \\
\hline \multirow{2}{*}{ RAVLT L $\neq$} & $\mathrm{C} / \mathrm{G}$ & $10.24(2.65)$ & $10.75(2.58)$ & 0.235 \\
\cline { 2 - 5 } & $\mathrm{PM} / \mathrm{C}$ & $11.36(2.24)$ & $12.15(2.26)$ & 0.007 \\
\hline \multirow{2}{*}{ FAS(X) $\neq$} & $\mathrm{C} / \mathrm{G}$ & $11.57(3.20)$ & $9.96(3.09)$ & 0.024 \\
\cline { 2 - 5 } & $\mathrm{PM} / \mathrm{C}$ & $10.24(2.73)$ & $11.78(4.06)$ & 0.023 \\
\hline
\end{tabular}

Table 3: Difference in groups' performance between baseline and follow up.

M (SD): Mean (Standard Deviation); C/G: Control Group; PM/G: Progressive Memory group; $p$ : 0.05; ‡: More points/better performance; $\dagger$ : Less Points/better performance; FRSSD: Functional Rating Scale for Dementia; RAVLT L: Rey Auditory Verbal Learning Test/learning ability; FAS(X): Verbal Fluency/letter X. 
Is it Possible the Training of Prospective Memory to Enhance Activities of Daily Living and Executive Function in People with Mild Cognitive Impairment? A Single-blind Randomized Controlled Trial

\section{Discussion}

Prospective memory refers to the ability to remember to perform a planned action or intention at a future time and is closely associated with working memory and executive function abilities $[25,40]$. Difficulties in PM are usually presented in people with MCI [41-43]. These difficulties have serious impact in quality of life [44]. Therefore, PM seems to be a crucial cognitive function for independent ADL [45].

Nowadays, it is well known that cognitive interventions are beneficial to some extent for people with MCI [43]. However, until now no data exist which assess the effectiveness of PM training both in PM, and executive function components in the same time, and also in ADL in people with aMCImd. Thus, the aim of the present controlled trial was to design and evaluate the impact of a new PM training program in the aforementioned abilities in people with aMCImd. The particular training of PM was chosen, as there is evidence that it is associated with the maintenance of independence and daily autonomy [42]. Moreover, since difficulties in PM and especially in the time-based prospective component are potentially early cognitive markers of dementia [46], we consider that cognitive programs which focus in PM training are ideal interventions for people with aMCImd, since they would minimize the possibility of conversion to AD.

According to our initial hypothesis, the $P M / G$ would present significant differences after the training session, compared to $\mathrm{C} / \mathrm{G}$ in PM and in executive function abilities. According to our results, significant difference between PM/G and C/G was noticed in working memory and in phonological verbal fluency but not in PM as expected. Specifically, at the follow up the PM/G outperformed the $\mathrm{C} / \mathrm{G}$ in the aforementioned abilities. Working memory and phonological verbal fluency, are considered to be parts of the executive function system $[47,48]$, which are practiced during the PM training.

Concerning working memory, it seems to be a significant predictor of complex prospective memory initiation [20], since it coordinates performance at multiple tasks, attends and inhibits other information, holds and manipulates information in memory [5]. PM failures are also associated with executive dysfunction presented in MCI. In this program we practiced executive functions and PM via the use of strategies and therefore, it makes sense that the PM/G at the end of intervention had better performance than $C / G$ at working memory, which is a prerequisite for successful PM [5].
On the other hand, regarding the statistical difference between $\mathrm{PM} / \mathrm{G}$ and $\mathrm{C} / \mathrm{G}$ in phonological verbal fluency, besides the fact that it is not clear the mechanism that associates phonological verbal fluency with PM it seems that verbal fluency is associated with executive function abilities especially in older adults. In order for a person to retrieve words at a task of phonemic verbal fluency, a) access to the mental lexicon, b) focus on the task, c) select words meeting certain constraints and, d) avoid repetition, are required $[49,50]$. Furthermore, we can't overlook that the ongoing tasks of the PM training in our study were tasks which comprised phonological or categorical verbal fluency tasks, which even though they differ from the cognitive tasks, it seems that the experimental group was familiar to the procedure. Therefore, the specific tasks seem to have played a crucial role to the $P M / G$ improvement in verbal fluency in comparison to the $\mathrm{C} / \mathrm{G}$.

Our results seem not to be in agreement with studies which support the idea that PM training is effective for people with aMCImd in improving only PM ability. Specifically, the study by Kinsella., et al. in 2009 [51], evaluated a 5-weeks PM intervention in 52 people with amnestic MCI. The program aimed to increase awareness on memory issues in daily life by using specific strategies. Their training program comprised strategies for improving organizational and attention skills, learning and remembering. Specific memory strategies for remembering specific everyday tasks were used (e.g., face-name recall or remembering to do things) - verbal categorization, elaboration, visual imagery, errorless learning and spaced retrieval. The neuropsychological assessment was performed at baseline, two weeks after the intervention and four months after the end of the intervention and comprised PM tests and questionnaires evaluating aspects of psychological well-being and metamemory. Their results concerning PM, showed that everyday memory, as was measured by performance on PM tasks, was increased compared to the waiting list controls. In contrast with their study, in our study a significant difference among experimental and control groups in PM tasks was not noticed, but difference appeared only in components of executive function. Therefore, our study also provides evidence that people with MCI possibly gain benefits from a well-structured PM training compared to $C / G$, but in specific executive function's abilities.

Regarding PM, the training failed to improve the PM ability in people with aMCImd. One possible explanation for this failure is associated with the RPA-ProMem Test which was used in order to assess PM. The aforementioned test is possibly not very easy or 
Is it Possible the Training of Prospective Memory to Enhance Activities of Daily Living and Executive Function in People with Mild Cognitive Impairment? A Single-blind Randomized Controlled Trial

reliable for assessing PM in people with aMCImd. Other researchers also noted that RPA-ProMem test is a difficult test to administer, especially the long-term PM tasks, in which the examined person has to complete the tasks outside of the memory clinic or the day care center [52]. On the contrary, self and informant-report questionnaires are possibly more useful, since they represent the individuals' own perspective [52]. Perhaps this test is not useful for measuring the progress of the deterioration.

According to our second hypothesis, the PM group at the end of the PM training would generalize the primary benefit yielded from the training in ADL. In accordance to our hypothesis, the PM/G showed improvement in the total score of the test assessing ADL. Besides the fact that we don't expect great difficulties in ADL in people with MCI, they seem to appear diminished functionality in specific aspects of ADL $[53,54]$. The fact that the ADL instrument being utilized in this study was a tool that assesses the caregiver's opinion, is very important since the difference in the functional status was obvious to caregivers. This result is very important, since the ultimate goal of cognitive training is to transfer the benefits yielded from training in ADL.

In agreement to our study, the study by Rose., et al. in 2015 [55], also administered a PM training program by using the Virtual Week (VW) computer game for a month. VW is a computerized game that goes through the course of a day on each circuit of the game dashboard, simulating the engagement in events and remembering to perform intended actions at appropriate times. For the need of the study, Rose at al. adjusted the VW computer game and the participants over the course of each virtual day had to perform different types of PM tasks (event-based tasks, time-based tasks). Many of the PM tasks simulated actual tasks, while the program comprised different task difficulty levels and each task's difficulty was increased over the 24 virtual days. Fifty-nine healthy older participants completed four (4) weeks of training, three (3) days per week. Training group was compared to a no-contact and an active control group. The active control group consisted of non-musicians older adults, engaged in a music training program in a classroom setting with a teacher. The participants were taught basic musical concepts such as rhythm, pitch, melody and voice. The program comprised motor, perceptual and cognitive tasks. The no-contact group comprised older adults who participated only in the pre-test and post-test sessions. The neuropsychological assessment was a battery of different PM paradigms, with different degrees of eco- logical validity, tests for working memory, processing speed, inhibition, fluid intelligence and ADL. At the end of the training, the participants in the Virtual Week group improved their performance in prospective memory and also in ADL [55]. However, compared to the aforementioned study, in our study an improvement in PM was not noticed. Two possible explanations are that a) the specific test we utilized for assessing PM was not appropriate and b) the duration of the training program was quite large since in the previous study the intervention lasted 4 weeks, whilst our PM program lasted for almost 6 months.

Another interesting finding of our study is that the PM/G after the completion of the PM program, enhanced abilities that were not directly practiced in the program such as episodic verbal learning. Verbal learning is an ability which requires preserved semantic learning strategies, since semantic structure is essential since the examinee has to actively cluster words of the same semantic group [56]. This procedure is demanded at verbal learning tasks such as in RAVLT. Therefore, verbal learning is closely related with executive functions. In our sessions the participants were taught specific strategies and techniques in order to manage to respond to the requires of the PM program. Among these strategies was the strategy of visual imagery, in which they taught and practiced how to use it during the first session. This strategy was able to make them to remember the time and the place in the future that they had to perform an obligation without using external aids such as calendars or mobile phones. Therefore, it makes sense that the PM/G increased in episodic verbal learning, since they started to use strategies and designed step by step new ways and solutions for their prospective memory problems. A similar study to ours, which was also based in a PM program utilizing visual imagery [27], tried to enhance PM abilities and to generalize the primary cognitive effect in other cognitive domains such as cognitive control (executive function) abilities in people with traumatic brain injury. The program comprised 10 weekly individual sessions lasting approximately 90 minutes each to a sample of 10 patients. The cognitive exercises which were administered via a computer screen, had different levels of complexity and were naturalistic in order to facilitate generalization and maintenance of the intervention effects. The intervention included five phases, which were the PM functioning understanding, the training to visualize simple images, the learning of visual imagery techniques and applying them in PM abilities and also in everyday conditions. According to their results the experimental group of traumatic brain injury reported less everyday PM failures 
Is it Possible the Training of Prospective Memory to Enhance Activities of Daily Living and Executive Function in People with Mild Cognitive Impairment? A Single-blind Randomized Controlled Trial

however the cognitive control tasks remained similar [27]. However, in our study, the visual imagery technique and the PM training succeeded to generalize the training's effect, not only in the domain of executive function such as phonological verbal fluency which was one of our basic goals, but also to transfer the training effect in other cognitive domains, such as episodic verbal memory, and to improve ADL.

The C/G showed cognitive and functional stability and a mild deterioration in phonological verbal fluency. MCI is a group characterized by a great heterogeneity [57], a percentage of which is expected to either progress to dementia with an annual MCI progression rate from 5 to $10 \%$ or to revert to normal cognition, whilst there is a number of people with MCI that remain stable [58]. The subtype is also playing a crucial role to the deterioration since people with aMCImd seem to be at high risk of progression. Therefore, our results regarding the stability and the mild deterioration of $\mathrm{C} / \mathrm{G}$ during the 6 months period was an expected finding.

\section{Limitations}

One major limitation of our study is the absence of a self-report questionnaire of everyday memory performance in order to assess the after-training subjective changes in PM performance. We consider that the self-report questionnaire would lead to more sufficient results and conclusions regarding the way that the PM program can assist people with aMCImd to improve their daily function and their self-esteem. Another limitation is the lack of more tools apart from RPA-ProMem in order to assess the effectiveness of our program to PM abilities. Based on the limitations that have already discussed, we consider that the use of other PM test would help us to clarify the effectiveness of this program to PM.

\section{Conclusion}

In this study we attempted to evaluate the effectiveness of a new designed PM training program focused in mental imagery strategy, in PM, executive function components and ADL, in people with MCI.

The PM training didn't succeed in improving the PM ability assessed only with RPA-ProMem test, however it helped people with aMCImd to improve executive functions, episodic memory and ADL. Therefore, it provides some evidence that PM programs are useful for people with aMCImd as they can improve cognitive domains related to executive functions. Furthermore, the PM program succeeded in improving ADL, which is always the ultimate goal of cognitive interventions for people with degenerative diseases.
The most important contribution of the implemented PM training program is that supports the idea that the process - based and the visual imagery strategy need to be combined with the PM training in order to generalize the cognitive effects in other domains. Moreover, the use of naturalistic prospective memory tasks is indispensable regarding the transfer of the primary effects in ADL. However, future studies on the efficacy of the specific program are needed, in order to provide more evidence on this field. Maybe it would be useful to design a study with a greater duration of cognitive training, or with more than one cognitive training programs since scientific data support the idea that more cognitive training is beneficial for people with MCI [59,60].

\section{Conflict of Interest}

The authors report no conflict of interest.

\section{Annex 1}

\section{Prospective memory $1^{\text {st }}$ level of training}

Task 1: Before the beginning of the session, remember to ask me to tell you a specific name.

PM target 1: The first target is to remember to tell me the name you heard as soon as you complete the session for today, that is before you leave the training room.

Visual imagery strategy: Before you continue the ongoing tasks try to follow some instructions for remembering the target that was given to you: imagine yourself to perform what has been asked from you, step by step.

- Formulate the image in your mind

- Write one by one in detail all the steps that you will follow.

Task 2: A supermarket leaflet and a shopping list will be given to you.

- You must calculate the total amount of money you will need in order to buy all the list's products.

- How much money would you pay more in the case that the products were without discount?

- List the products according to the alphabetical order and categorize them..

PM target 2: In 20 minutes you have to stop the ongoing task either you have completed it or not and come to tell me what you ate yesterday for dinner. Then you must give the green ball that 
Is it Possible the Training of Prospective Memory to Enhance Activities of Daily Living and Executive Function in People with Mild Cognitive Impairment? A Single-blind Randomized Controlled Trial

you will find at the training room to the person that is sitting next to you.

The same procedure is followed concerning the visual imagery strategy.

The 20 minutes start as soon as the ongoing task begins.

Task 3: The next ongoing task comprises anagrammatisms, phonological fluency tasks, and tasks of abstract thinking. You must complete these tasks as better as you can.

PM target 3: During the ongoing task you will hear the sound of a bell. At the second bell that you will hear, you must ask me to give you a blue pen. Afterwards, you will continue completing the tasks, until you hear the bell sound ringing for the fourth time. Then you should ask me to give you the red pen, in order to use it for the rest of the tasks.

The same procedure is followed concerning the visual imagery strategy.

The 20 minutes start as soon as the ongoing task begins.

\section{Bibliography}

1. Einstein Gilles $\mathrm{O}$ and Mark A McDaniel. "Normal aging and prospective memory". Journal of Experimental Psychology: Learning, Memory, and Cognition 16.4 (1990): 717.

2. McDaniel Mark A and Gilles O Einstein. "Strategic and automatic processes in prospective memory retrieval: A multiprocess framework". Applied Cognitive Psychology: The Official Journal of the Society for Applied Research in Memory and Cognition 14.7 (2000): S127-S144.

3. Rendell Peter G., et al. "Prospective memory in multiple sclerosis". Journal of the International Neuropsychological Society: JINS 13.3 (2007): 410.

4. Ellis Judi and Lia Kvavilashvili. "Prospective memory in 2000: Past, present, and future directions". Applied Cognitive Psychology: The Official Journal of the Society for Applied Research in Memory and Cognition 14.7 (2000): S1-S9.

5. Kliegel Matthias., et al. "Complex prospective memory and executive control of working memory: A process model". Psychological Test and Assessment Modeling 44.2 (2002): 303.

6. Dagenais Emmanuelle., et al. "Role of executive functions in prospective memory in multiple sclerosis: Impact of the strength of cue-action association". Journal of Clinical and Experimental Neuropsychology 38.1 (2016): 127-140.
7. Costa Alberto., et al. "Prospective memory impairment in mild cognitive impairment: An analytical review". Neuropsychology Review 21.4 (2011): 390-404.

8. Baddeley Alan., et al. "Dual-task performance in dysexecutive and nondysexecutive patients with a frontal lesion". Neuropsychology 11.2 (1997): 187.

9. Kliegel Matthias., et al. "Prospective memory in older adults: where we are now and what is next". Gerontology 62.4 (2016): 459-466.

10. Henry Julie D., et al. "A meta-analytic review of prospective memory and aging". Psychology and aging 19.1 (2004): 27.

11. Hernandez Cardenache, Rene., et al. "Evaluating different aspects of prospective memory in amnestic and nonamnestic mild cognitive impairment". International Scholarly Research Notices 2014 (2014).

12. Zhou Ting., et al. "Deficits in retrospective and prospective components underlying prospective memory tasks in amnestic mild cognitive impairment". Behavioral and Brain Functions 8.1 (2012): 39.

13. Blanco-Campal., et al. "Detection of prospective memory deficits in mild cognitive impairment of suspected Alzheimer's disease etiology using a novel event-based prospective memory task". Journal of the International Neuropsychological Society 15 (2009): 154-159.

14. Spíndola Lívia and Sonia Maria Dozzi Brucki. "Prospective memory in Alzheimer's disease and Mild Cognitive Impairment". Dementia and Neuropsychologia 5.2 (2011): 64-68.

15. Shelton Jill Talley., et al. "Improving prospective memory in healthy older adults and individuals with very mild Alzheimer's disease". Journal of the American Geriatrics Society 64.6 (2016): 1307-1312.

16. Baldo Juliana V and Arthur P Shimamura. "Letter and category fluency in patients with frontal lobe lesions". Neuropsychology 12.2 (1998): 259.

17. Schwartz Sophie and Juliana Baldo. "Distinct patterns of word retrieval in right and left frontal lobe patients: a multidimensional perspective". Neuropsychologia 39.11 (2001): 12091217.

18. Mousavi Shokoufeh., et al. "Evaluation and comparison of episodic memory in elderly people: the effect of strategies education on memory reinforcement". Clinical Social Work and Health Intervention 2017a 8 (2017): 59-66. 
19. Burkard Christina., et al. "Is working memory necessary for implementation intentions to enhance prospective memory in older adults with cognitive problems?". Journal of Applied Research in Memory and Cognition 3.1 (2014): 37-43.

20. Kliegel Matthias., et al. "Plan formation, retention, and execution in prospective memory: A new approach and age-related effects". Memory and Cognition 28.6 (2000): 1041-1049.

21. Albert Marilyn S., et al. "The diagnosis of mild cognitive impairment due to Alzheimer's disease: recommendations from the National Institute on Aging-Alzheimer's Association workgroups on diagnostic guidelines for Alzheimer's disease". Focus 11.1 (2013): 96-106.

22. Petersen Ronald C and John C Morris. "Mild cognitive impairment as a clinical entity and treatment target". Archives of neurology 62.7 (2005): 1160-1163.

23. Rose Nathan S., et al. "Age and individual differences in prospective memory during a" Virtual Week": The roles of working memory, vigilance, task regularity, and cue focality". Psychology and aging 25.3 (2010): 595.

24. McDaniel Mark A and Julie M Bugg. "Journal of Applied Research in Memory and Cognition". Journal of Applied Research in Memory and Cognition 1 (2012): 45-50.

25. Hering Alexandra., et al. "Prospective memory training in older adults and its relevance for successful aging". Psychological research 78.6 (2014): 892-904.

26. Cavallini Elena., et al. "Aging and everyday memory: the beneficial effect of memory training". Archives of gerontology and geriatrics 37.3 (2003): 241-257.

27. Potvin Marie-Julie., et al. "Prospective memory rehabilitation based on visual imagery techniques". Neuropsychological Rehabilitation 21.6 (2011): 899-924.

28. Petersen Ronald C. "Mild cognitive impairment as a diagnostic entity”. Journal of internal medicine 256.3 (2004): 183-194.

29. Radford Kylie A., et al. "Validation of a new measure of prospective memory: the Royal Prince Alfred Prospective Memory Test". The Clinical Neuropsychologist 25.1 (2011): 127-140.

30. McDaniel Mark A., and Michael K. Scullin. "Implementation intention encoding does not automatize prospective memory responding". Memory and cognition 38.2 (2010): 221-232.

31. Morris David M., et al. "Constraint-induced movement therapy for motor recovery after stroke". Neuro Rehabilitation 9.1 (1997): 29-43.
32. Folstein Marshal F., et al. "Mini-mental state": a practical method for grading the cognitive state of patients for the clinician". Journal of Psychiatric Research 12.3 (1975): 189-198.

33. Fountoulakis Konstantinos N., et al. "Mini mental state examination (MMSE): a validation study in Greece". American Journal of Alzheimer's Disease and Other Dementias ${ }^{\circledR} 15.6$ (2000): 342-345.

34. Hutton JT., Instrumental Activities of Daily Living (IADL) Scale. "Alzheimer's Disease in Conn's Current Therapy". Psychopharmacology Bulletin 24 (1990): 778-781.

35. Kounti Fotini., et al. "Functional cognitive assessment scale (FUCAS): a new scale to assess executive cognitive function in daily life activities in patients with dementia and mild cognitive impairment". Human Psychopharmacology: Clinical and Experimental 21.5 (2006): 305-311.

36. Rey Andŕe. "L'examen clinique en psychologie". (1958).

37. Messinis Lambros., et al. "Age and education adjusted normative data and discriminative validity for Rey's Auditory Verbal Learning Test in the elderly Greek population". Journal of Clinical and Experimental Neuropsychology 38.1 (2016): 23-39.

38. Kosmidis Mary H., et al. "The verbal fluency task in the Greek population: Normative data, and clustering and switching strategies". Journal of the International Neuropsychological Society: JINS 10.2 (2004): 164.

39. SPSS, IBM. "Statistics for Windows [Computer Program]. Version 23.0". (2015).

40. Schnitzspahn Katharina M., et al. "The role of shifting, updating, and inhibition in prospective memory performance in young and older adults". Developmental Psychology 49.8 (2013): 1544.

41. Kinsella Glynda J., et al. "Mild cognitive impairment and prospective memory: Translating the evidence into neuropsychological practice". The Clinical Neuropsychologist 32.5 (2018): 960-980.

42. Pereira Antonina., et al. "Enhancing prospective memory in mild cognitive impairment: The role of enactment". Journal of Clinical and Experimental Neuropsychology 37.8 (2015): 863877.

43. Sherman Dale S., et al. "The efficacy of cognitive intervention in mild cognitive impairment (MCI): a meta-analysis of outcomes on neuropsychological measures". Neuropsychology Review 27.4 (2017): 440-484. 
44. Woods Steven Paul., et al. "Does prospective memory influence quality of life in community-dwelling older adults?". Aging, Neuropsychology, and Cognition 22.6 (2015): 679-692.

45. Hering Alexandra., et al. "Prospective memory is a key predictor of functional independence in older adults". Journal of the International Neuropsychological Society 24.6 (2018): 640645.

46. Hsu Yen-Hsuan., et al. "Prospective Memory in Subjective Cognitive Decline". Alzheimer Disease and Associated Disorders 29.3 (2015): 229-235.

47. Beaty Roger E and Daniel L Schacter. "Episodic Memory and Cognitive Control: Contributions to Creative Idea Production". The Cambridge Handbook of the Neuroscience of Creativity (2018): 249.

48. Abwender David A., et al. "Qualitative analysis of verbal fluency output: Review and comparison of several scoring methods". Assessment 8.3 (2001): 323-338.

49. Shao Zeshu., et al. "What do verbal fluency tasks measure? Predictors of verbal fluency performance in older adults". Frontiers in Psychology 5 (2014): 772.

50. Fisk John E and Charles A Sharp. "Age-related impairment in executive functioning: Updating, inhibition, shifting, and access". Journal of Clinical and Experimental Neuropsychology 26.7 (2004): 874-890.

51. Kinsella Glynda J., et al. "Early intervention for mild cognitive impairment: a randomised controlled trial". Journal of Neurology, Neurosurgery and Psychiatry 80.7 (2009): 730-736.

52. Man David WK., et al. "Validation of the Cambridge prospective memory test (Hong Kong Chinese version) for people with stroke". Neuropsychological Rehabilitation 25.6 (2015): 895-912.

53. Bangen Katherine J., et al. "Complex activities of daily living vary by mild cognitive impairment subtype". Journal of the International Neuropsychological Society 16.4 (2010): 630-639.

54. Farias Sarah T., et al. "MCI is associated with deficits in everyday functioning". Alzheimer disease and associated disorders 20.4 (2006): 217.

55. Rose Nathan S., et al. "Cognitive and neural plasticity in older adults' prospective memory following training with the Virtual Week computer game". Frontiers in Human Neuroscience 9 (2015): 592.
56. Ribeiro Filipa., et al. "Verbal learning and memory deficits in mild cognitive impairment". Journal of Clinical and Experimental Neuropsychology 29.2 (2007): 187-197.

57. Gillis Cai., et al. "The incidence of mild cognitive impairment: A systematic review and data synthesis". Alzheimer's and Dementia: Diagnosis, Assessment and Disease Monitoring 11 (2019): 248-256.

58. Pandya Seema Y., et al. "Does mild cognitive impairment always lead to dementia? A review". Journal of the Neurological Sciences 369 (2016): 57-62.

59. Tsolaki Magda., et al. "Effectiveness of nonpharmacological approaches in patients with mild cognitive impairment". Neurodegenerative Diseases 8.3 (2011): 138-145.

60. Eleni Poptsi., et al. "Use it more and keep it alive: a Longitudinal Randomized Controlled Trial in people with Mild Cognitive Impairment". Hellenic Journal of Nuclear Medicine 20.2 (2017): 218-232.

\section{Assets from publication with us}

- Prompt Acknowledgement after receiving the article

- Thorough Double blinded peer review

- Rapid Publication

- Issue of Publication Certificate

- High visibility of your Published work

Website: www.actascientific.com/

Submit Article: www.actascientific.com/submission.php Email us: editor@actascientific.com

Contact us: +919182824667

Citation: Christina Agogiatou, et al. "Is it Possible the Training of Prospective Memory to Enhance Activities of Daily Living and Executive Function in People with Mild Cognitive Impairment? A Single-blind Randomized Controlled Trial”. Acta Scientific Medical Sciences 4.10 (2020): $102-113$. 